\title{
Evaluation of Tomato Juice Quality Using Surface Enhanced Raman Spectroscopy
}

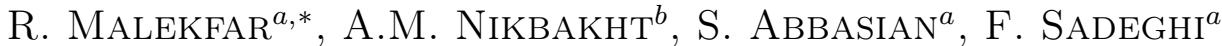 \\ AND M. MOZAFFARI ${ }^{a}$ \\ ${ }^{a}$ Faculty of Basic Sciences, Tarbiat Modares University, Tehran P.O. Box 14115-175, I.R. Iran \\ ${ }^{b}$ Faculty of Agriculture, Tarbiat Modares University, Tehran P.O. Box 14115-336, I.R. Iran
}

(Received March 15, 2010)

\begin{abstract}
Tomato (Lycopersicon esculentum L.) is a valuable fruit cultivated in the world as an important plant. There are a lot of industries engaged with the drying, processing, quality control and sorting of tomato. A rapid and reliable measurement of qualitative factors of tomato and its products can be a serious necessity for the producers and industrialists. The objective of this research was to implement SERS as a modern technique to evaluate some qualitative parameters of tomato juice. To prevent the effect of cartenoids and florescence background, the juices were centrifuged. Silver colloid was prepared according to Lee and Meisel method for SERS experiments. The results were compared with the spectra obtained from ordinary spontaneous Raman spectroscopy technique data. Three major bands assigned to carbohydrates in tomato were detected and analyzed. Moreover, a medium band related to proteins could be identified. Comparison of SERS spectra with the normal spontaneous Raman spectra proved that SERS has a privilege in the measurement of carbohydrates and proteins as an effective, rapid and precise technique.
\end{abstract}

PACS numbers: 78.30.-j, 33.20.Fb

\section{Introduction}

Raman spectroscopy has an important role in the identification and characterization of molecular structures. The Raman scattering cross-section is inherently rather weak, which prevents the possibility of achieving low detection limit with normal Raman spectroscopy. Surface Enhanced Raman Spectroscopy, (SERS) is a much more sensitive and selective technique to detect low-concentration analytes. SERS was initially observed in 1974 by Fleischman et al. [1-3]. They reported strong Raman scattering from pyridine adsorbed on silver electrode surfaces that had been roughened electrochemically by oxidation-reduction cycles. There are different kinds of SERS-active substrates used in the wide range of SERS applications such as metal colloids, coated filter papers with metal nanoparticles, electrochemically roughened metal electrodes etc. [4-6]. In order to put in a nutshell, when the molecule adsorbed on the surface of a noble metal such as gold and silver, more frequencies can be observed compared to the normal Raman spectroscopy. Among the metals, silver is the best choice because of its small imaginary part of the dielectric constant and higher thermal conductivity [7-9]. Silver nanoparticles due to their vast applications in many fields such as electromagnetic interference shielding, antibiocial medical device and surface enhanced Raman scattering have been of great importance in recent decades.

Tomato (Lycopersicon esculentum L.) is a significant fruit in the global agricultural market due to its wide

\footnotetext{
* corresponding author; e-mail: Malekfar@Modares.ac.ir
}

use in food industry both as fresh consumption and the extensive processed products. The fruit is mainly composed of water, soluble and insoluble solids, organic acids (mostly citric acid) and micronutrients such as carotenoids and vitamins A and C [10]. Several quality criteria are considered for tomato, from which Soluble Solid Content (SSC), acidity (pH) and color are the most important parameters affecting the consumers' appreciation for selection [11].

Most of the instrumental techniques to measure quality parameters of fruits are destructive and involve a considerable amount of manual work. However, researches have presently been focused on developing nondestructive techniques, e.g. Visible/NIR spectroscopy, Raman spectroscopy, etc. for measuring fruit quality attributes [12].

In Raman spectroscopic investigations there arise two main problems. First, the low scattering cross-section of biological samples, which results in low signal levels, and second, the excitation of fluorescence can often be observed, which makes it difficult to obtain Raman spectra and calibrate them with the constituents. SERS is known for its sensitivity towards a wide range of biological active compounds.

The objective of this research was to present results of micro-Raman and SERS studies on tomato fruits. We investigated the chemical composition of the fruits by the spectra recorded by the normal spontaneous Raman and SERS techniques.

\section{Experimental procedure}

Tomato samples of special varieties were collected and juiced for the spectroscopic tests. Silver colloid was prepared according to Lee and Meisel's method. Briefly, $18 \mathrm{mg}$ of $\mathrm{AgNO}_{3}$ was dissolved in $100 \mathrm{ml}$ distilled wa- 
ter and the solution was heated to boiling. Then $7 \mathrm{ml}$ of $1 \%$ trisodium citrate aqueous solution was added into the boiling silver nitrate solution at once, under vigorous stirring. The mixed solution was kept boiling for a further 8 minutes. Finally, the green-gray solution was obtained which was stable for several days or weeks [4]. The absorbance spectrum was recorded by PG-Instruments-T80 spectrophotometer. A Zeta Sizer Nano-ZS instrument was employed to obtain size distributions of silver nanoparticles. The mixing ratio, tomato juice to silver colloid, is an important factor for the enhancement of spectra in SERS. The maximum enhancement occurs when the target molecule covers thoroughly the whole surface of nanoparticles. As there was no reference for mixing ratios of tomato and colloid, several ratios were tested using trial and error method.

In Raman spectroscopy, three major bands dominate the spectrum due to cartenoids, while the bands of interest related to carbohydrates and proteins appear as medium and weak bands. Therefore, the cartenoids were extracted from the juices using centrifuging method. Tomato juices were centrifuged at $1000 \mathrm{rpm}$ using SIGMA 4 K 15 refrigerated centrifuge. The consequence is removing the coloring composition from the juice, remaining carbohydrates which are soluble in water.

Sugars (fructose and glucose) were determined using a HPLC (WATERS 600, E USA) equipped with a BondapackTM C18 $(250 \mathrm{~mm} \times 4.6 \mathrm{~mm})$ column as a separator and a Waters refractive index detector. Mobile phase was Acetonitrile:Water (75:25), at $1 \mathrm{ml} / \mathrm{min}$. Sigma-Aldrich standards were used for building calibration curves. Tomato juices were centrifuged at $1000 \mathrm{rpm}$ using SIGMA 4 K 15 refrigerated centrifuge and twice filtered to be completely clarified and injected at the injection volume $20 \mu \mathrm{l}$.

Raman and SERS spectra on the samples were obtained using the Nicolet Almega Dispersive Raman (Thermo Scientific, USA) spectrometer consisting a laser operating at the maximum power of $100 \mathrm{~mW}$ at $532 \mathrm{~nm}$. The laser output power of $30 \mathrm{~mW}$ used for analysis was low enough to prevent possible laser induced sample damage yet provided a high signal to noise ratio. Data were collected at $2 \mathrm{~cm}^{-1}$ resolution with 16 scans. Spectra were obtained in the Raman shift range between 100 and $4000 \mathrm{~cm}^{-1}$. The system was operated using Omnic software (Version 5.1, Madison, WI).

\section{Results and discussions}

Silver colloidal was characterized by UV/Vis/NIR spectrophotometer and Dynamic Light Scattering (DLS). Figure 1 shows the surface plasmon absorption band of silver colloidal around the $430 \mathrm{~nm}$. The distribution of particles size of Ag metal that was shown in Fig. 2 is relatively narrow and the average particle size is about $16.9 \mathrm{~nm}$.

Figure 3 demonstrates the SERS spectra of different mixing ratios from 1:2, in the bottom, to $1: 8$, in the top, of juice and colloid, respectively. By using the ratios $1: 1$ and 1:2, there appeared no specific band, while some

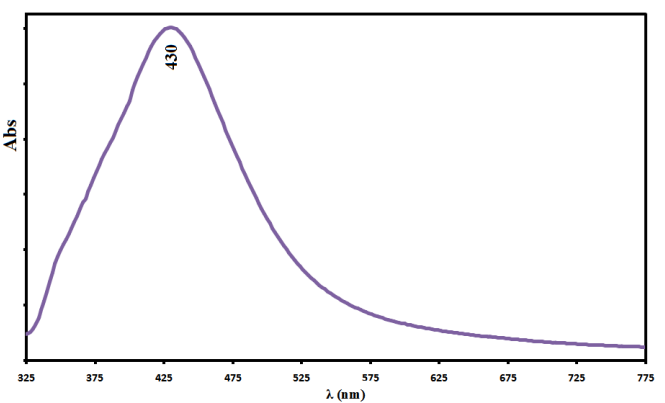

Fig. 1. UV/Vis Spectrum of the prepared Ag colloidal sample.

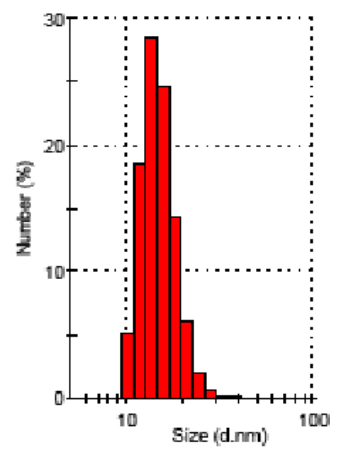

Fig. 2. The particle size distribution of a typical prepared Ag colloidal sample.

medium peaks could be distinguished at the ratio of $1: 3$. By increasing the colloid portion could improve the spectra up to the mixing ration of 1:6 (Fig. 3). Beyond this limit, the peaks start to disappear again. Therefore, the ratio of 1:6 was selected as an optimum level for mixing tomato juice with the silver colloid.

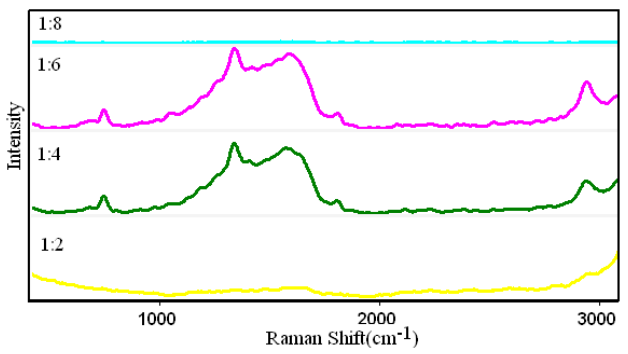

Fig. 3. SERS spectra of tomato juice at different mixing ratios of tomato juice: silver colloid.

As the first phase of the experiments, the juice of fruits was investigated by SERS technique. The major peaks which observed were mainly related to the cartenoids (i.e. lycopene and carotene) which produced undesirable background fluorescence.

As shown in Fig. 4, there can be no clear interpretation of the juice spectrum. Although, some bands relating to cartenoids and even weak assignments to carbohydrate 


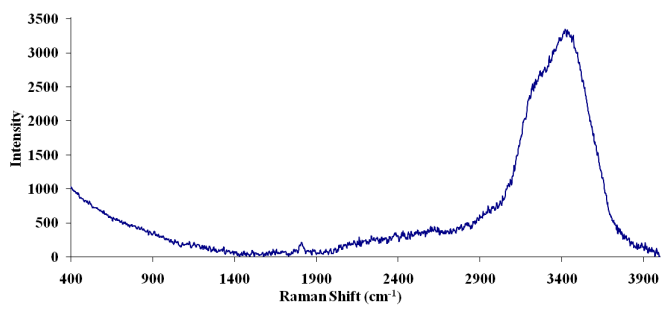

Fig. 4. SERS spectrum of un-centrifuged juice.

can be observed, the dominating bands together with a noisy region prohibit the spectrum analysis and quantitative investigation.

As a sequence, the second phase of experiments were conducted on the centrifuged juices excluding cartenoids to investigate some medium and weak bands present in the SERS spectra of tomato fruit (Fig. 5b). The band resulting from $\mathrm{C}-\mathrm{C}$ and $\mathrm{C}-\mathrm{O}$ stretching vibrations of carbohydrates was observed at $738 \mathrm{~cm}^{-1}$. This is an important band in Raman spectrum hidden by cartenoids which can be dominated the whole region of $700-1600 \mathrm{~cm}^{-1}$ [11]. According to liquid chromatography experiments, carried out to determine the exact type of sugars in the samples, it was revealed that a medium tomato $(100 \mathrm{~g})$ fruit contains 3-4 g carbohydrates, mostly as fructose and glucose. Thus we can reliably assign the carbohydrates to sugars in tomato fruit, which is of great value as an effective and practical technique to measure the sugars of tomato and its Brix value (soluble solids content).

The peak observed at $1333 \mathrm{~cm}^{-1}$ in Fig. $5 \mathrm{~b}$ is related to $\mathrm{CH}_{2}$ vibration of $\alpha$-glucose in tomato fruit [13]. Figure 5a presents a typical Raman spectrum of centrifuged juice. As shown in Fig. 5a, none of the mentioned peaks were observed in the normal spontaneous Raman technique spectrum. Another $\mathrm{C}-\mathrm{H}$ stretching vibration at $2930 \mathrm{~cm}^{-1}$ is highly correlated with the carbohydrates, mainly glucose and fructose, in tomato fruit [14]. Additionally, the medium and weak bands observed at $1580 \mathrm{~cm}^{-1}$ can be assigned to proteins [11].

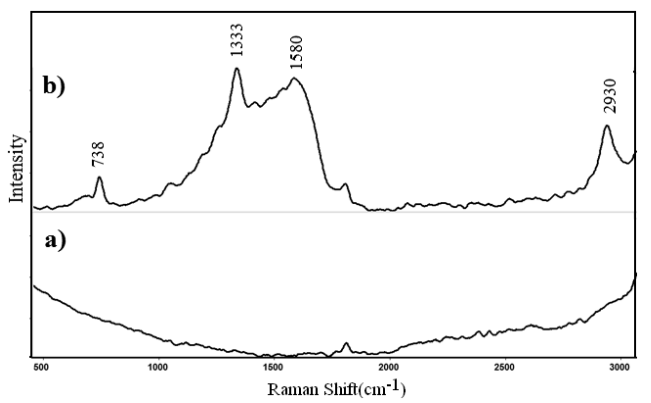

Fig. 5. (a) The normal spontaneous Raman spectrum of centrifuged juice; (b) the SERS spectrum of the mixture of centrifuged juice and silver colloidal solution.
Thus, SERS proved to be an advantageous method to evaluate major parameters of tomato fruit in comparison with the normal spontaneous Raman spectroscopy. The method is suitable for application in quality control lines and fruit processing industries.

\section{Conclusions}

Some qualitative parameters of tomato juice are detected by Surface Enhanced Raman Spectroscopy. The fruits were juiced and centrifuged to eliminate the effect of pigments and cartenoids, since the presence of these constituents prohibits the clear interpretation and analysis of the spectra. Silver colloidal was used as a SERS substrate and characterized by UV/Vis/NIR spectrophotometer and Dynamic Light Scattering technique. The results showed that by using SERS method, the major bands at $738 \mathrm{~cm}^{-1}, 1333 \mathrm{~cm}^{-1}$ and $2930 \mathrm{~cm}^{-1}$ could be assigned to the carbohydrates in tomato fruit. The chromatography tests also revealed that the main carbohydrates in tomato are sugars which have great importance to be detected. Furthermore, a medium band related to proteins could be distinguished. Comparing to the normal spontaneous Raman spectra on tomato juice, SERS had an advantage in measuring the qualitative parameters of the fruit. Therefore, it is highly suggested to be applied in control lines and fruit processing industries.

\section{References}

[1] C. Jing, Y. Fang, J. Colloid Interface Sci. 314, 46 (2007).

[2] Z. Niu, Y. Fang, J. Colloid Interface Sci. 303, 224 (2006).

[3] D. Wu, Y. Fang, J. Colloid Interface Sci. 265, 234 (2003).

[4] X. Zhang, N.C. Shah, R.P. Van Duyne, Vibrational Spectroscopy 42, 2 (2006).

[5] T. Vo-Dinh, Trends Analyt. Chem. 17, 557 (1998).

[6] E. Smith, G. Dent, Modern Raman Spectroscopy, a Practical Approach (2006).

[7] L.R. Wang, Y. Fang, Spectrochim. Acta 63, 614 (2006).

[8] D.G. Yu, W.C. Lin, C.H. Lin, b, L.M. Chang, M.C. Yang, Mater. Chem. Phys. 101, 93 (2007).

[9] G. Xiao, S. Man, Chem. Phys. Lett. 447, 305 (2007).

[10] A.M.K. Pedro, M.M.C. Ferreira, Analyt. Chim. Acta 595, 221 (2007).

[11] H. Schulz, M. Baranska, Vib. Spectrosc. 43, 13 (2007).

[12] J. Dijkstra, F. Ariese, C. Gooijer, U.A.Th. Brinkman, Trends Anal. Chem. 24, 304 (2005).

[13] J. Twardowski, P. Anzenbacher, Raman and IR Spectroscopy in Biology and Biochemistry Polish Scientific Publishers PWN Ltd, Warsaw 1994, p. 269.

[14] M.M. Paradkar, J. Irudayaraj, S. Sakhamuri, Appl. Eng. Agr. 18, 379 (2002). 